\title{
Understanding effects of obstructive sleep apnoea and its treatment on the brain and autonomic regulation needs further research
}

\author{
Esther I. Schwarz ${ }^{1}$, Christopher D. Turnbull ${ }^{2,3}$, John R. Stradling ${ }^{2,3}$, Malcolm Kohler $^{1}$ \\ ${ }^{1}$ Department of Pulmonology and Sleep Disorders Center, University Hospital of Zurich, Zurich, Switzerland; ${ }^{2}$ NIHR Oxford Biomedical Research \\ Centre, University of Oxford, Oxford, UK; ${ }^{3}$ Oxford Centre for Respiratory Medicine, Oxford University Hospitals NHS Foundation Trust, Oxford, UK \\ Correspondence to: Esther I. Schwarz. Department of Pulmonology and Sleep Disorders Center, University Hospital of Zurich, Zurich, Switzerland. \\ Email: EstherIrene.Schwarz@usz.ch. \\ Response to: Jaster JH. Reperfusion injury to ischemic medullary brain nuclei after stopping continuous positive airway pressure-induced CO2-reduced \\ vasoconstriction in sleep apnea. J Thorac Dis 2018;10:S2029-31.
}

Submitted Jun 27, 2018. Accepted for publication Jul 08, 2018.

doi: $10.21037 /$ jtd.2018.07.51

View this article at: http://dx.doi.org/10.21037/jtd.2018.07.51

We thank Dr. Jaster for reading our articles with interest and sharing his thoughts on the potential impacts of untreated obstructive sleep apnoea (OSA) and continuous positive airway pressure (CPAP) effects on medullary brain nuclei. There are three topics we would like to address: (I) the hypothesis of an association between OSA and ischemic pathology of medullary brain nuclei and a potential exacerbation by CPAP, (II) the different roles of $\mathrm{pCO}_{2}$ in OSA and central sleep apnoea (CSA), and (III) the effects of a short-term CPAP withdrawal on the brain and the heart.

The view's expressed by Dr. Jaster are interesting and challenging hypotheses, but so far not underlined by convincing scientific evidence. We couldn't find any evidence for an independent association of ischaemic lesions of the solitary tract nuclei and OSA in the cited references of the author, which are letters and comments and not original research data. The main statement by the author was that untreated sleep apnoea results in medullary brain ischemia, which may be exacerbated by both CPAP and its withdrawal as a consequence of $\mathrm{CO}_{2}$-mediated cerebral vasoconstriction and vasodilation.

Advisement 1: autoregulation of ventilation is impaired in case of medullary pathology (e.g., stroke), and this predisposes to both sleep-disordered breathing and breathing disturbances during wakefulness. However, when thinking of a cause-effect-relationship, there is no convincing evidence that OSA results in ischemiainduced changes of medullary nuclei due to perfusion or oxygenation impairment of the medulla. In view of the findings of a recent CPAP withdrawal trial demonstrating severe nocturnal cerebral hypoxemia in some patients with severe untreated OSA (1), the role of nocturnal medullary tissue hypoxia might be an interesting target to study to better understand autonomic instability/derangements and respiratory and cardiac control in OSA.

Advisement 2: the SAVE trial and the SERVE-HF trial are very important randomised controlled trials. The SAVE trial showed that CPAP had no effect on secondary prevention in patients with OSA and cardioand cerebrovascular disease, and the SERVE-HF trial showed that adaptive servo-ventilation led to increased cardiovascular mortality in patients with CSA/CheyneStokes Breathing in heart failure. These trials leave many questions unanswered and many have speculated on potential mechanisms that might explain these rather unexpected findings-indeed the hypothesis of Dr. Jaster could be further elucidated in patients with CSA on assisted servo-ventilation. However, the two trials included two pathophysiological distinct forms of sleep disordered breathing-OSA and CSA. Despite the known effects of $\mathrm{PaCO}_{2}$ changes on cerebral vascular tone, the effects of OSA on cerebral autoregulation seem to be more complex and not only affected by $\mathrm{PaCO}_{2}$. We agree that the potential effects of positive airway pressure and especially invasive mechanical ventilation on cerebral perfusion as consequences of changes in $\mathrm{PaCO}_{2}$ and haemodynamic 
have to be kept in mind in specific patient groups. Studying patients with treatment-emergent CSA while being treated with CPAP for OSA might give further insight into the topics raised by Dr. Jaster.

Advisement 3: OSA is usually undiagnosed and untreated for several years and CPAP is often not used over the whole night. It is unlikely that changes to the mentioned nuclei tract will occur within two weeks of untreated OSA or that CPAP withdrawal causes a reperfusion injury to the medulla oblongata. It is also unlikely that medullary changes explain any of the consequences found in response to OSA recurrence in short-term CPAP withdrawal. Several randomised controlled trials have demonstrated the safety of a short-term CPAP withdrawal in both populations with and without cardio- and cerebrovascular disease $(2,3)$. We found that CPAP withdrawal results in a pronounced increase in blood pressure and other consequences of untreated OSA, which include both persistent and cycling nocturnal cerebral tissue oxygenation. However, we also have observed that CPAP withdrawal neither affects myocardial (1) nor cerebral endothelial function during wakefulness.

Overall, the hypotheses expressed here are of interest and changes in the medulla oblongata could be addressed in

Cite this article as: Schwarz EI, Turnbull CD, Stradling JR, Kohler M. Understanding effects of obstructive sleep apnoea and its treatment on the brain and autonomic regulation needs further research. J Thorac Dis 2018;10(8):E664-E665. doi: $10.21037 /$ jtd.2018.07.51 future physiological and clinical trials in OSA.

\section{Acknowledgements}

None.

\section{Footnote}

Conflicts of Interest: The authors have no conflicts of interest to declare.

\section{References}

1. Schwarz EI, Furian M, Schlatzer C, et al. Nocturnal cerebral hypoxia in obstructive sleep apnoea: a randomised controlled trial. Eur Respir J 2018;51.

2. Kohler M, Stoewhas AC, Ayers L, et al. Effects of continuous positive airway pressure therapy withdrawal in patients with obstructive sleep apnea: a randomized controlled trial. Am J Respir Crit Care Med 2011;184:1192-9.

3. Schwarz EI, Schlatzer C, Stehli J, et al. Effect of CPAP Withdrawal on myocardial perfusion in OSA: A randomized controlled trial. Respirology 2016;21:1126-33. 